Cite this article: Roulet J-F, Abdulhameed N, Shen C.

Reinforcement of polymethylmethacrylate (PMMA) resin with perforated meta plates. Stoma Edu J. 2015;2(2):100-107.

\title{
REINFORCEMENT OF
}

POLYMETHYLMETHACRYLATE

\section{(PMMA) RESIN WITH PERFORATED METAL PLATES}

https://doi.org/10.25241/stomaeduj.2015.2(2).art.1

\section{Jean-François Roulet ${ }^{1 a}$ Nader Abdulhameed ${ }^{1 b}$ Chiayi Shen ${ }^{1 c}$}

'Department of Restorative Dental Sciences College of Dentistry, University of Florida Gainesville FL32610-0415, USA

a. DDS, PhD, Prof hc, Professor Director of Center for Dental Biomaterials b. BDS, MS, Visiting scientist, Candidate PhD Material Sciences c. PhD, Associate Professor

Received: November $27^{\text {th }}, 2015$ Accepted: December $4^{\text {th }}, 2015$

\section{* Corresponding author:} Professor Jean-Francois Roulet, DDS, PhD, Prof hc

Director of Center for Dental Biomaterials

Department of Restorative Dental Sciences College of Dentistry, University of Florida 1395 Center Drive, Room D9-6

PO Box 100415, Gainesville FL 32610-0415, USA Tel / Fax: (352) 273-5850 e-mail:JRoulet@dental.ufl.edu

\section{Abstract}

Background: Full dentures show a high rate of fractures under clinical use. Therefore strengthening of the denture base is expected to be beneficial with respect to reducing the fracture risk. Preformed perforated thin metal plates seem to be a simple tool for reinforcement.

Objective: To test the strengthening effect of perforated metal plates incorporated into a PMMA based resin as a function of the bonding strategy. Materials and Methods: Preformed gold plated stainless steel grid strengtheners were purchased, pressed flat and embedded in wax and processed as dentures using the Ivobase hybrid injection moldable material (Ivoclar Vivadent, Schaan, Liechtenstein). Prior to processing the surfaces were either not treated or treated with a primer (Monobond Plus, Ivoclar Vivadent, Schaan, Liechtenstein) and grit blasted and primed. They were then sectioned into beams for three point bending test. Resin beams of identical dimensions without reinforcement were used as control. Flexural strength and modulus were calculated based on the load to fracture determined by an Instron universal testing machine. The data were analyzed with ANOVA and Tukey's test.

Results: Flexural strength: control beams fractured at $78.8 \pm 5.9 \mathrm{MPa}$, while, with the exception of the grit blasted samples, the experimental groups showed significantly higher strength (97.2 respect. $95.4 \mathrm{MPa}$ ).

Flexural Modulus: The control group yielded $2261.7 \pm 261.4 \mathrm{MPa}$, while all experimental groups had a significantly higher modulus (3239.1 - 3952.4 MPa)

The surface treatments did not show significant differences.

Conclusions: The grid strengtheners tested had a significant effect on strengthening. Under the conditions of this study, surface priming did not increase the mechanical properties of the reinforced bars.

Keywords: denture, reinforcement, injection molding process, three point bending test

\section{Introduction}

The shift from vulcanized rubber to polymethacrylate resin (PMMA) has dramatically improved the esthetics of removable dental prostheses. After its invention 1 it took several years until it became usable for manufacturing individually formed pieces by reducing its polymerization shrinkage of $21 \%$ to a few percentages by using spherical pre polymerized particles. ${ }^{2}$ These particles which come as a powder could be mixed with liquid MMA and were processed into a dough, that could be used to process dentures of high esthetic appearances. However, being a rather brittle material with modest mechanical properties ${ }^{3}$ to make it a material, it was still far away from fulfilling the mechanical requirements for an ideal material for dentures. ${ }^{4}$ The result is that full dentures are prone to fractures, which occur in stress concentration areas such as a large frennel notch ${ }^{5}$, mainly due to fatigue. ${ }^{6}$ Very thin areas, poor fitting dentures and a lack of balanced occlusion are additional factors increasing the fracture risk. In the late 90's the British Dental Practice Board reported that the cost of repairing annually 1.2 million dentures is 18 million $\mathrm{f} 7$. Typically, the ratio of upper to lower denture fractures is about 2:1 with the most common 


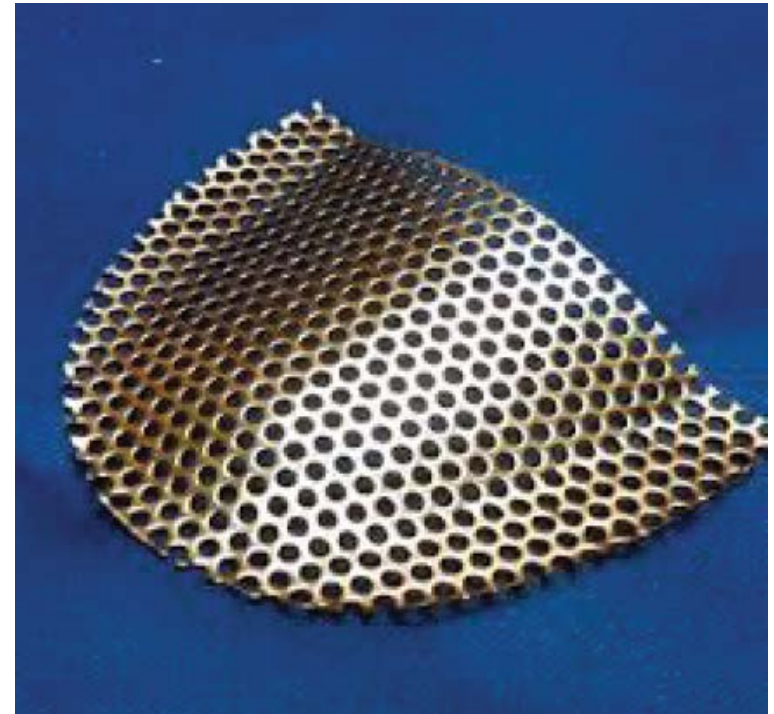

Figure 1. Dentaurum Strengthening Grid

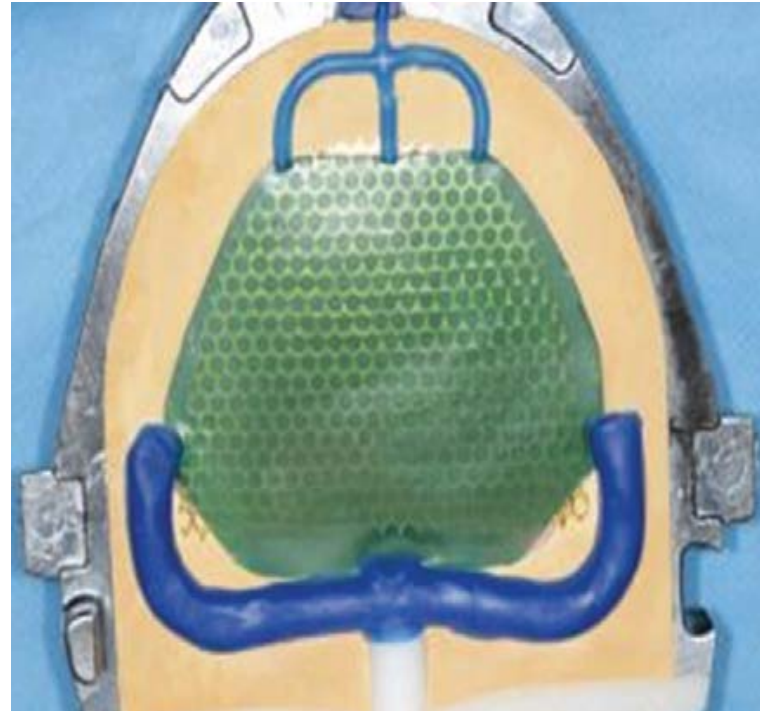

Figure 2. Wax/grit strengthener plate in injection molding flask with sprues.

Arrows show reposition areas without wax covering the grid

Studies investigating reinforcement with mesh and a braided wire plate did not report a significant improvement in the transverse strength of acrylic. However, incorporating silanated glass fibers into acrylic resin improved the fracture strength. Vallittu et al $1994^{14}$ found a linear relationship for the increase. The more fibers were incorporated the greater the increase in strength.

Metal reinforcement could fail at the resin/ strengthener interface since areas of stress concentration occur around embedded materials. Various approaches have been used to improve the adhesion between the metal surface and acrylic resin such as sandblasting, silanization and metal adhesive resins. The effect of the metal strengthener's surface roughness on the fracture resistance of the acrylic denture base material was investigated by Vallittu (1992). ${ }^{15}$ The investigation showed that the surface roughening of the metal wires used to reinforce the acrylic resin denture base material increased the fracture resistance of the test specimens. The best results were achieved by sandblasting..$^{15}$ In Europe, preformed perforated gold plated steel plates (Grid strengthener, Dentaurum GmbH \&Co, KG, Ispringen, Germany) (Fig.1) are sold as strengthener for full dentures, which simplify the incorporation into dentures. However the manufacturer does not give any instructions how to treat the surface before being incorporated into the PMMA resin.

Based on literature data, one would expect a strengthening effect, if such plates were bonded to the resin. Therefore the purpose of the study was to test the strengthening effect of the perforated metal plates as a function of the bonding strategy. The null hypotheses were as follows:

1. The metal grid strengthener does not strengthen acrylic resin.

2. The different surface treatments do not affect strengthening. 
Tukey Test Mean For Flexural Strength

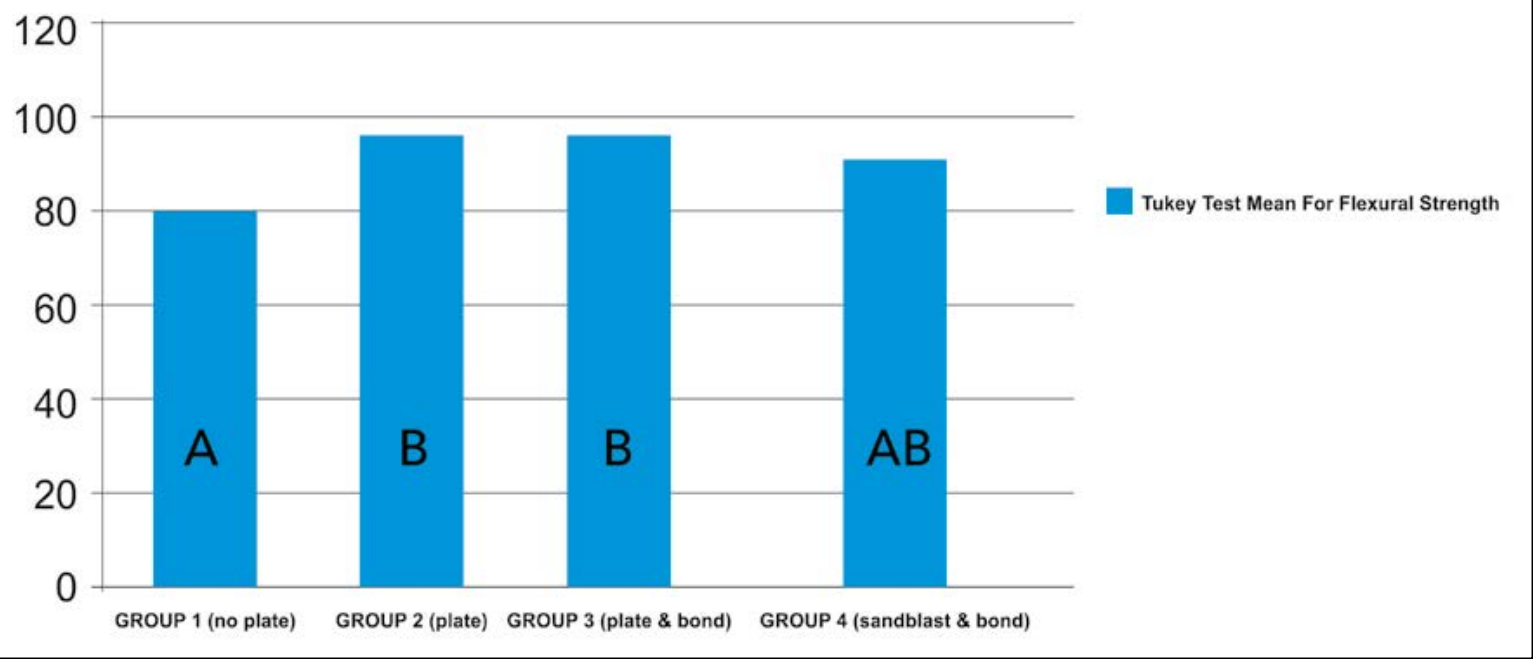

Figure 3. Flexural Strength. $P<0.05$, same letter $=$ same statistical group

\section{Materials and Methods}

Nine Dentaurum Grid Strengthener $10.4 \mathrm{~mm}$ stainless steel, gold plated with perforations $(\varnothing$ 2.5mm, Article No. 318-104-00, Dentaurum GmbH \&Co, KG, Ispringen, Germany) were purchased and pressed in a hydraulic laboratory press (Carver Lab press, Wabash, IN, USA) at $9800 \mathrm{~N}$ for 2 days until flat. Using wax plates of different thicknesses (Truwax, USA) (0.5 mm for bottom, $1.6 \mathrm{~mm}$ for top) "sandwiches" of $2.5 \mathrm{~mm}$ thickness were produced, positioning the metal plate at $0.5 \mathrm{~mm}$ from the bottom side.

At 3 peripheral sites the wax was removed, so the samples could be repositioned into the flask after boiling out of the wax. Using flasks for the injection technique (IvoBase, Ivoclar Vivadent, Schaan, Liechtenstein) and the appropriate spruing (Fig. 2) and yellow microstone, type III (Whip Mix, Louisville, KY, USA) a two part form was created, which allowed a defined 3D reposition of the grid before injecting the resin.

For control purposes wax plates $2.5 \mathrm{~mm}$ thick were embedded as described above.

Three plates per group were conditioned the following way before being repositioned into the flask:

Group 1: No grid strengthener (control).

Group 2: No surface treatment of grid strengthener Group 3: Monobond Plus (Ivoclar Vivadent) ${ }^{16}$ was applied to grid strengthener with paint brush, let react for $30 \mathrm{~s}$ and then the solvent was evaporated for $10 \mathrm{~s}$ by blowing with an air syringe.

Group 4: Grid strengthener was grit blasted with $\mathrm{Al}_{2} \mathrm{O}_{3} 100 \mu \mathrm{m}$ at $0.25 \mathrm{MPa}$ for $10 \mathrm{~s}$, then Monobond Plus was applied as described above.

The next step was to injection mold the Ivobase hybrid material (Ivoclar Vivadent) using the Ivomat Polymerization unit with program \#1 for 45 minutes. After removing from the flasks the resin/metal plates and the control plates were sectioned with a diamond saw (IsoMet 1000 Precision Cutter, Buehler, Lake Bludd, IL USA) under water cooling into approximately $10 \mathrm{~mm} \times 75 \mathrm{~mm} \times 2.5 \mathrm{~mm}$ beams.

For testing purposes the thickness and width of every beam was measured individually using a caliper (Model 06-664-16, Fisher Scientific, Pittsburgh, PA, USA). The yield was 4 beams /plate which produced 12 beams per group. The beams were stored in water for 7 days prior to perform the mechanical testing.

The beams were subjected to a 3-point bending test according to ISO standard 1567 at $5 \mathrm{~mm} / \mathrm{min}$. The fracture strength was calculated using the following formula:

$$
\sigma=\frac{3 F L}{2 w h^{2}}
$$

The Flexural Modulus was calculated as well according to:

$$
E=\frac{L^{3} F}{4 w h^{3} d}
$$

- $\sigma$ is the flexural strength

- $F$ is the load at fracture or peak load in strength (specimen embedded with mesh did not break)

- $L$ is the span between the two supports

- $w$ is the width of the specimen

- $h$ is the height (or thickness) of the specimen

- $d$ is the deflection of the specimen due to the load $F$ Data were analyzed with an ANOVA (SAS, Cary, NC, USA) and multiple pairwise comparisons were performed with the Tukey's HSD test.

\section{Results}

Table 1, Fig. 3 and Fig. 4 show that incorporation of grid strengthener improves both the flexural strength and modulus of the denture base resin. ANOVA shows that there is statistically significant 


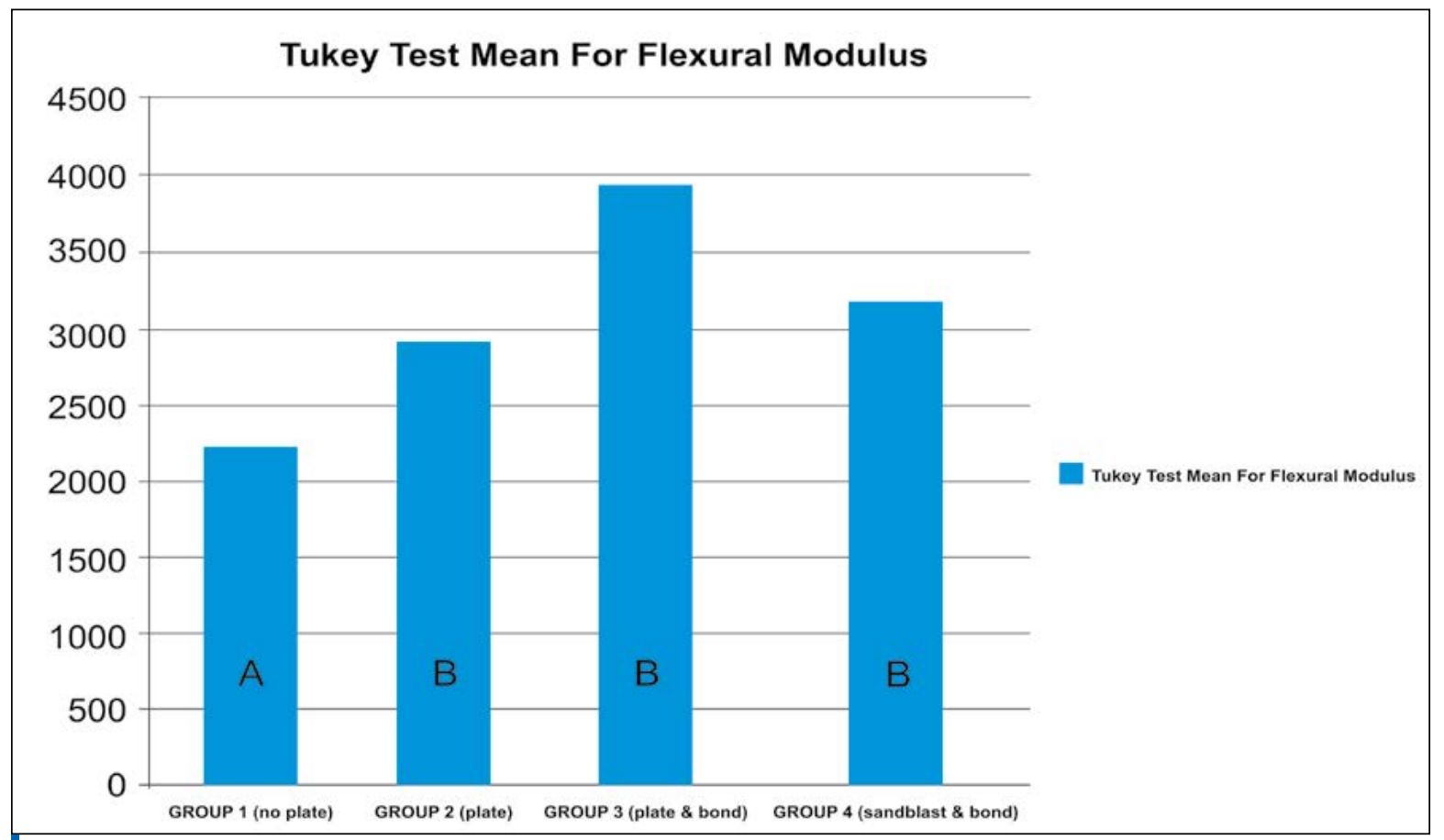

Figure 4. Flexural Modulus $p<0.05$, same letter = same statistical group

Table 1 Results for Flexural Strength and Flexural Modulus

\begin{tabular}{|l|c|c|c|c|}
\hline \multicolumn{1}{|c|}{ Groups } & Fracture Strength (MPa) & SD & Fracture Modulus (MPa) & SD \\
\hline Group 1 C. & $78.8^{\mathrm{A}}$ & 5.9 & $2261.7^{\circ}$ & 261.4 \\
\hline Group 2: GS & $97.2^{\mathrm{B}}$ & 14.1 & $3477.9^{\circ}$ & 770.6 \\
\hline Group 3: GS, MP & $95.4^{\mathrm{B}}$ & 19.3 & $3952.4^{\circ}$ & 268.5 \\
\hline Group 4: GS, GB , MP & $91.7^{\mathrm{AB}}$ & 15.5 & $3239.1^{\circ}$ & 366.6 \\
\hline p-value (ANOVA) & 0.03 & & $<0.0001$ & \\
\hline
\end{tabular}

$\mathrm{C}=$ control, $\mathrm{GS}=$ grid strengthener, $\mathrm{MP}=$ Monobond Plus, $\mathrm{GB}=$ grit blasted

difference among the four group of specimens in flexural strength $(p=0.0137)$ and flexural modulus $(p<0.0001)$. Tukey's HSD test shows that there is no statistical difference among the three grid strengthener containing groups, and there is no statistical difference between the control and the group reinforced with gritblasted strengthener and bonding agent.

For flexural modulus, all three groups with grid strengthener are significantly higher than that of the control, and additional surface treatment of the strengthener did not result in additive strengthening of the denture base.

Thus the first null hypothesis can be rejected, which is not the case for the second null hypothesis, which must be partially accepted.

\section{Discussion}

The purpose of the study was to investigate if the grid strengthener had a strengthening effect on the flexural strength and modulus of a PMMA based denture base resin and should additional surface treatment, such as roughening and bonding, enhance these properties. Three point bending test was chosen, because its test geometry represents the closest the load situation in a denture in a patients mouth.

The grids were positioned close to the bottom of the bars ( $\sim 0.5 \mathrm{~mm}$ ), where the bar is under tension during testing and fractures of the denture resin were expected.

Thus it could be expected to have the best possible strengthening of a metal plate to a PMMA structure.

The injection molding technique was chosen, because it allowed better precision in positioning the metal grids within the PMMA plate and the resin injection does not subject strengthener to vertical forces during processing keeping the grid to stay in place.

Traditional compressive molding technique, which requires multiple trial flasking, would 
likely cause drift of the grid during processing. Previous studies have shown that fibers or mesh without surface modification would not render the denture base any strengthening effect. Some studies even showed negative effect. Substantial improvement of strengthening was observed only when the surface of the strengthener fiber or mesh was gritblasted, coating of bonding agent or combination of the two. ${ }^{17}$

The results of this study showed basically that the metal grid alone had a statistically significant strengthening effect in flexural strength and modulus.

However, the improvement of flexural strength from the grids receiving gritblasting and coating with bonding agent (Group 4) is not statistically significantly from the control (Group 1 ).

Contrary to previous studies, the results show that there is no statistical difference among the groups comprising strengtheners. In other words, grit blasting and coating of bonding agent provided no additional benefit in enhancing flexural strength and modulus.

Grit blasting is a well-recognized method of surface roughening that leads to bonding enhancement. In addition, the bonding agent used in the study is an ethanol solution known to consist of three distinct compounds; namely, silane methacrylate, phosphoric acid methacrylate and disulphide methacrylate. ${ }^{16}$

They are designed to bond to three different surfaces: silane targeting silica (not present in the test groups), a phosphate containing methacrylate focusing on metal oxides (in this case chromium oxides) and a disulfide methacrylate aiming for gold.

The cause of inability of the bonding agent and grit blasting to enhance flexure strength and modulus should be clarified.

When glass fibers in the form of woven, loose and continuous fiber bundles were first investigated for reinforcing PMMA denture base resin, they were either mixed discretely with the PMMA dough or by lamination with glass cloth. ${ }^{18}$

The addition of fibers did not give a substantial improvement to the tensile strength caused by the lacking of adhesion between the polymer matrix and the untreated fibers.

These fibers acted as inclusion bodies in the PMMA resin mixture that actually weakened the resin instead of strengthening.

Treating glass fiber with silane compounds before incorporating in the PMMA dough presented a potential of strengthening PMMA. ${ }^{19}$

If the fibers were made into cloth form instead of loose form, untreated cloth form fibers had demonstrated strengthening effect whereas untreated loose glass fibers had a weakening effect. ${ }^{17}$

Using metallic inserts in reinforcing PMMA denture base resin yielded similar results, where embedding metal fibers weakened the polymer due to poor adhesion between the acrylic resin matrix and the metal fiber inserts. ${ }^{17}$

Gritblasting ${ }^{17}$, silanization of the metallic insert ${ }^{20}$, and metal bonding adhesive resin ${ }^{21}$ have been reported to improve the adhesion between the metal surface and acrylic resin.

It is of interest to observe that untreated metal grid strengthened the acrylic resin but no additional improvement was realized when the metal grid had been gritblasted or coated with bonding adhesive for metal surface.

This unexpected result could only be caused by the design of the grid.

Metal grid used in this study is a perforated stainless steel with staggered pattern of $\varnothing 2.5 \mathrm{~mm}$ holes and $1.25 \mathrm{~mm}$ between adjacent perforations. When this grid is incorporated during the processing described earlier, the resin fills the perforated spaces (Fig. 2).

The result is an interpenetrating structure between the metal grid and denture resin that interlocks the resin and grid mechanically.

Therefore, as the resin component is being stressed during testing, the metallic component will be subjected to the same stress simultaneously. In other words, metal grid strengthener exhibits strengthening effect without the need of bonding between the metal grid and the resin.

Although the study shows that resultant bonding from gritblasting and adhesive coating would not improve the flexure strength and modulus of the denture base resin, microleakage can still occur from lack of bonding or poor bonding between the denture base resin and the cast metal framework of a removable partial denture.

It can cause discoloration and staining of the margins at the metal-resin interface over time. ${ }^{22}$

Therefore, the need of a durable bonding between the metal grid and the denture resin remains critical with respect to staining over time.

Should that type of bond be achieved by gritblasting, adhesive resin or combination of the two should be investigated further by additional in vitro studies with specimens after a long-term storage in appropriate solutions or through longterm clinical observation.

The presence of a metal grid within a denture base resin qualifies the specimen design in this study as a composite structure and the mechanical property of the final structure will depend on those of the metal grid and the resin.

It implies that there may be a most appropriate design of perforated metal for strengthening denture base resins.

When filler particles or fibers are used as strengthener, the rule of mixtures, which is a weighted mean used to predict various properties of a composite material, can be used to estimate the strength and stiffness of the test specimens used in the study.

However, depending on the design, such as the size, pattern and distribution of the perforation, thickness of the metal grid and the direction of force applied, the effective strength and the 
stiffness of the metal grid vary.

Finding such metal grid experimentally may not be the most efficient approach. A finite element analysis with a well-defined model, can be used to demonstrate stress distribution within a metal grid reinforced denture base.

The model can be modified to reflect the change in design and the site or direction of loading. The design the most favorable stress distribution can then be selected and tested experimentally.

\section{Conclusions}

Within the limitation of this study, we can conclude that:

1. Metal grid as-received without modification by sandblast or adhesive resin coating can strengthen the denture base resin in the flexure strength and modulus.

2. Surface modification of the metal grid is still needed as a mean of preventing discoloration or staining at the metal/resin interface.

\section{Acknowledgement}

The authors thank Dr. Jennifer Santos for allowing the publication of this paper.

This experiment has been performed as requirement for acquiring the Specialist Degree in Prosthodontics of Dr. J. Santos. 


\section{Bibliography}

1. Röhm 0, Bauer W. Geformte Kunststoffe aus Polyacrylsäure in ihren Verbindungen oder Mischungen. 1928; Patent Nr. DE656421.

2. Röhm 0, Trommersdorf E. Verfahren zum polymerisieren von Vinyl-, Acryl-, und Methacrylverbindungen. 1935; Patent Nr. DE747596.

3. Gieler CW, Skinner EW. Physical properties of some of the newer denture plastics. J Dent Res. 1939;18(4):381-387.

4. Jagger DC, Harrison A, Jandt KD. The reinforcement of dentures. J Oral Rehabil. 1999;26(3):185-194. Review.

5. Rees JS, Huggett R, Harrison A. Finite element analysis of the stress-concentrating effect of fraenal notches in complete dentures. Int J Prosthodont. 1990;3(3):238-240.

6. Johnston EP, Nicholls JI, Smith DE. Flexure fatigue of 10 commonly used denture base resins. J Prosthet Dent. 1981;46(5):478-483.

7. Dental Practice Board. Dental Practice Board annual report. Eastbourne, UK 1997.

8. Beyli MS, von Fraunhofer JA. An analysis of causes of fracture of acrylic resin dentures. J Prosthet Dent. 1981;46(3):238-241. 9. Darbar UR, Huggett R, Harrison A. Denture fracture--a survey. Br Dent J. 1994;176(9):342-345.

10. Hargreaves AS. The prevalence of fractured dentures. A survey. Br Dent J. 1969;126(10):451-455.

11. Schneider RL. Diagnosing functional complete denture fractures. J Prothet Dent. 1985; 54(6):809-814.

12. Jagger $D C$, Harrison $A$. The fractured denture-solving the problem. J Primary Dent Care 1998;5:159-162.
13. Smith DC. The acrylic denture: Mechanical evaluation midline fracture. Br Dent J. 1961;110:257-267.

14. Vallittu PK, Lassila VP, Lappalaineri R. Acrrylic resin-fiber composite-Part I: The effect of fiber concentration on fracture resistance. J Prosthet Dent. 1994;71(6):607-612.

15. Vallittu PK, Lassila VP. Effect of metal strengthener's surface roughness on fracture resistance of acrylic denture base material. J Oral Rehabil. 1992;19(4):385-391.

16. Völkel T. Make it easy to yourself! Monobond Plus Scientific Documentation. Ivoclar Vivadent, Research and Development, Schaan, Liechtenstein 2011.

17. Jagger $D C$, Harrison $A$, Jandt KD. The reinforcement of dentures. J Oral Rehabil. 1999;26(3): 185-194. Review.

18. Smith DC. The non-metallic denture base-recent developments. Dent Pract Dent Rec. 1957;8(11):73-80.

19. Solnit GS. The effect of methyl methacrylate reinforcement with silane-treated and untreated glass fibers. J Prosthet Dent. 1991;66(3):310-314.

20. Vallittu PK. Effect of some properties of metal strengtheners on the fracture resistance of acrylic denture base material. J Oral Rehabil. 1993;20(3): 241-248.

21. Polyzois GL. Reinforcement of denture acrylic resin: the effect of metal inserts and denture resin type on fracture resistance. Eur J Prosthodont Restor Dent. 1995;3(6):275-278. 22. Shimizu H., Takahashi Y. Review of adhesive techniques used in removable prosthodontic practice. J Oral Sci. 2012;54(3):205-211. 


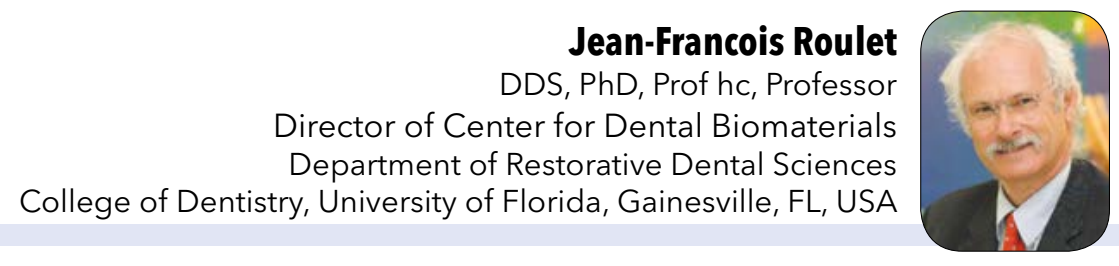

Jean-François Roulet, DDS, Dr med dent, PhD, is the former chair and current professor of the Department of Restorative Dental Sciences at the University

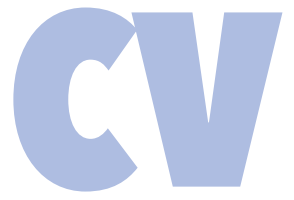
of Florida. Dr Roulet has published more than 180 research papers as well as reviews, book chapters, and books.

He also mentored more than 150 theses. He is a renowned international lecturer with over 800 appearances to date.

Dr. Roulet is member of many professional organizations, has won numerous awards, and holds four patents.

He was editor of The Journal of Adhesive Dentistry and Oral Health \&

Preventative Dentistry. Since 1996 he is editor of Prophylaxe Impuls. His areas of interest include minimally invasive dentistry, dental materials (i.e., composites and ceramics), adhesive dentistry, esthetic dentistry, and application concepts in preventive dentistry.

\section{Perforated thin metal plates incorporated into a PMMA based resin:}

$\square$ a. Are more resistant to fracture;

b. Are easily fractured;

c. Only priming can avoid fracturing;

$\square \mathrm{d}$. The grid does not affect resistance to fracture.

\section{The Flexural Modulus of the plate is affected by:}

$\square$ a. Only the thickness of the plate;

$\square$ b. The quality of the resin and of the polymerization method;

$\square$ c. Priming the grid and the type of resin employed;

$\square$ d. Not affected by any factor.

\section{What categories of resins are recommended for the prosthetic plate?}

$\square$ a. Simple thermopolymerizable resins;

b. Simple baropolymerizable resins;

c. Injectable flexile resins;

$\checkmark$ d. (High impact) injectable baropolymerizable resins.

\section{When is the ideal prosthetic plate obtained?}

$\square$ a. When the metal grid strengthener is used;

$\square$ b. When the grid is not primed;

c. When simple thermopolymerizable resins are used;

$\square \mathrm{d}$. When the polymerization regime is not observed. 